\title{
Article \\ Properties of Quantizer and Dequantizer Operators for Qudit States and Parametric Down-Conversion
}

\author{
Peter Adam 1,2, Vladimir A. Andreev ${ }^{3}$, Margarita A. Man'ko ${ }^{3, *}$, Vladimir I. Man'ko ${ }^{3,4,5,6}$ and Matyas Mechler ${ }^{2} \mathbb{C}$ \\ 1 Institute for Solid State Physics and Optics, Wigner Research Center for Physics, P.O. Box 49, \\ H-1525 Budapest, Hungary; adam.peter@wigner.mta.hu \\ 2 Institute of Physics, University of Pécs, Ifjúság útja 6, H-7624 Pécs, Hungary; mechler@fizika.ttk.pte.hu \\ 3 Lebedev Physical Institute, Leninskii Prospect 53, 119991 Moscow, Russia; andrvlad@yandex.ru (V.A.A.); \\ mankovi@lebedev.ru (V.I.M.) \\ 4 Moscow Institute of Physics and Technology, State University, Institutskii Per. 9, Dolgoprudnyi, \\ 141700 Moscow, Russia \\ 5 Russian Quantum Center, Skolkovo, 143025 Moscow, Russia \\ 6 Department of Physics, Tomsk State University, Lenin Avenue 36, 634050 Tomsk, Russia \\ * Correspondence: mankoma@lebedev.ru
}

check for

updates

Citation: Adam, P.; Andreev, V.A.; Man'ko, M.A.; Man'ko, V.I.; Mechler, M. Properties of Quantizer and Dequantizer Operators for Qudit States and Parametric DownConversion. Symmetry 2021, 13, 131 https://doi.org/10.3390/sym1301 0131

Received: 31 December 2020 Accepted: 12 January 2021 Published: 14 January 2021

Publisher's Note: MDPI stays neutral with regard to jurisdictional clai$\mathrm{ms}$ in published maps and institutional affiliations.

Copyright: $\odot 2021$ by the authors. Licensee MDPI, Basel, Switzerland. This article is an open access article distributed under the terms and conditions of the Creative Commons Attribution (CC BY) license (https:// creativecommons.org/licenses/by/ $4.0 /)$.

\begin{abstract}
We review the method of quantizers and dequantizers to construct an invertible map of the density operators onto functions including probability distributions and discuss in detail examples of qubit and qutrit states. The biphoton states existing in the process of parametric down-conversion are studied in the probability representation of quantum mechanics.
\end{abstract}

Keywords: quantum tomography; probability representation; quantizer; dequantizer; qubit

\section{Introduction}

Quantum states are usually determined using the wave functions $[1,2]$ or the density matrices [3,4]. However, there exists the other way to describe quantum states. For systems with continuous variables such as oscillators, quantum states are associated, e.g., with Wigner functions [5] or Husimi-Kano functions [6,7]. In connection with the development of theories for laser photon states, quasidistributions describing these states were introduced by Glauber [8] and Sudarshan [9]. For systems with discrete variables such as spin systems and $\mathrm{N}$-level atom systems, qubits, and qudits, the Wigner functions were introduced and studied; see, e.g., [10,11]. All the various methods of describing the system quantum states are related to different experimental possibilities to study physical properties of the systems, which are available in some states characterized by a density operator acting on the Hilbert space.

Recently, the probability representation of quantum states for continuous variables [12] and discrete spin variables [13-18] was suggested; see review [19] and recent papers [20-33]. All mentioned descriptions of quantum states can be constructed, using different methods of mapping the density operators and state vectors onto different kinds of functions, including the functions which are the probability distributions associated with measurements of various physical characteristics of quantum systems; the functions are called symbols of the density operators. If these functions are functions of finite number of discrete variables, we call these variables the symbol components.

The aim of this paper is to present a review of the approach to employ different representations of quantum states. The representations can be constructed using some sets of variables, discrete or continuous. Sometimes these variables can have a concrete physical meaning [34]. To describe quantum states, one can also select observables which can be measured. There are various ways to introduce such numerical characteristics determining quantum states. A method of finding the sets of variables specifying the quantum state is the use of operators called quantizers and dequantizers. Using these operators, one 
can find a set of variables which determines the quantum state. To do this, one should calculate the trace of the product of dequantizers with the quantum-state density operator. The resulting sets of variables, discrete or continuous ones, form a function called the state symbol. Operators which form sets of quantizers and dequantizers have a certain algebraic structure. Different sets of quantizers and dequantizers give different symbols of the state density operators. These symbols may have different physical meanings.

On the other hand, some algebraic structures can be associated with each set of quantizer-dequantizer operators, in particular, they can have the properties of either the associative algebras or Lie algebras. It turns out that the requirement that the symbols of the state density operators have the physical meaning imposes additional conditions on these algebras. In this paper, we consider systems of qudits of an arbitrary dimension $d$ as physical objects. Such an object can exist as a multilevel system. The density matrix of such a system state is the $d \times d$ matrix; it includes $d^{2}-1$ independent parameters, and different physical meanings can be given to these parameters. In [22-24], the interpretation of qutrits was proposed as a set of qubits interacting with each other.

In [31], this approach was generalized to qudits of an arbitrary dimension. Representing a qudit as a set of qubits, one can interpret their parameters either as probabilities of the values of spin projections on certain axes or as the mean values of these projections. The example to construct the probability representation of qudit states on the base of $S U(2)$ symmetry was given in [25] including examples of qutrit and ququart. In [27], we considered qubits and qudits; their representation was found in terms of the probabilities and mean values. We demonstrated that these sets of probabilities and mean values form the representation spaces of symmetry groups corresponding to the density matrices; for the qubit case, these representations were found explicitly.

In $[21,22]$, we constructed associative algebras and Lie algebras that are generated by such quantizer-dequantizer operators and showed that in some cases the structure constants of these algebras have additional symmetry properties. We established that all quantizers and dequantizers whose symbols are measurable observables, such as spinprojection probabilities and mean values, are Hermitian operators and presented the algebraic structure of such sets. We constructed the associative algebras and Lie algebras generated by the quantizer-dequantizer operators and calculated the structure constants of these algebras [21,22]. In the case where the symbol of the density operator corresponds to the mean values of spin projections, we showed that the corresponding dequantizers and quantizers form a self-dual system, i.e., their operators coincide up to a numerical multiplier. The structure constants of the Lie algebras generated have an additional property of symmetry. Also in [21,22], we introduced a concept of the inner product of the symbol components, which is dual to star product of symbols, and demonstrated that it can be used to construct the associative algebras and Lie algebras, with elements being the components of symbols of the density operators.

In this paper, we consider in detail qudits of an arbitrary dimension $d$; for them, the sets of quantizers and dequantizers are found, for which the components of the corresponding symbols have the meaning either of probabilities or mean values. In view of the application of the approach, we consider, as an example, the process of parametric downconversion $[35,36]$ and discuss in the probability representation the qutrit and ququart states realized in this process.

This paper is organized as follows.

In Section 2, we consider general properties of quantizer and dequantizer operators and describe invertible maps of the operators acting in a Hilbert space onto functions called symbols of the operators. Particular examples of the quantizer-dequantizer operators for the system of qudits are presented in Section 3. In Section 4, we discuss in detail the example of qutrit states and study the system of four-level atoms and its states in the probability representation of quantum mechanics. We discuss the possibility to apply the quantizerdequantizer approach for studying such a nonlinear phenomenon as the parametric downconversion in Section 5. Finally, we present our conclusions and prospectives in Section 6. 


\section{The Method of Quantizers and Dequantizers}

The method of quantizer-dequantizer operators was suggested in [37]; see also [38-41], to describe the construction of quantum symplectic tomographic-probability distributions [12] identified with quantum states as well as other possible quasidistribution functions such as the Wigner function [5] describing the photon (and other) states. This method is based on the idea to introduce Wigner functions for spin states using the notion of quantizer operator [42]. The method looks as follows.

Let us consider the set of operators $\hat{U}(x)$ acting on a Hilbert space $\mathcal{H}$. The operators of this set depend on parameters $x=\left(x_{1}, x_{2}, \ldots, x_{N}\right)$, which can be either continuous or discrete ones. For any operator $\hat{A}$ acting on the Hilbert space $\mathcal{H}$, we introduce its symbol $f_{A}(x)$, which is the function defined, in view of the dequantizer operators, as

$$
f_{A}(x)=\operatorname{Tr}(\hat{A} \hat{U}(x)) .
$$

We assume that there exists the other set of operators $\hat{D}(x)$ acting in the Hilbert space $\mathcal{H}$ and called the quantizer operators; these operators provide the possibility to reconstruct the operator $\hat{A}$ if the symbol of this operator is known, using the relation

$$
\hat{A}=\int f_{A}(x) \hat{D}(x) d x
$$

This formula is written for the case of continuous parameters $x=\left(x_{1}, x_{2}, \ldots, x_{N}\right)$. If the parameters are discrete ones, the integral in (2) is replaced by the corresponding sum.

The condition for the existence of a pair of the quantizer-dequantizer operators is given by the equality

$$
\operatorname{Tr}(\hat{A} \hat{U}(x))=\int \operatorname{Tr}\left(\hat{A} \hat{U}\left(x^{\prime}\right)\right) \operatorname{Tr}\left(\hat{U}(x) \hat{D}\left(x^{\prime}\right)\right) d x^{\prime} ;
$$

a particular case of this relation takes place if the following equality is valid:

$$
\operatorname{Tr}\left(\hat{U}(x) \hat{D}\left(x^{\prime}\right)\right)=\delta\left(x-x^{\prime}\right) .
$$

In the case of discrete variables $x$, the integrals in the above equation are replaced by the sum over the discrete indices.

For the dequantizer operators satisfying the equality $\hat{U}^{+}(x)=\hat{U}(x)$ and the condition $\operatorname{Tr} \hat{U}(x)=1$ along with the constraint $\hat{U}(x) \geq 0$, the dequantizer operators have all the properties of density operators; this means that, if operators $\hat{A}$ are taken to be density operators $\hat{\rho}$ of a system state, the function

$$
P(x)=\operatorname{Tr}(\hat{\rho} \hat{U}(x)) \geq 0
$$

is, according to Born's rule, the probability distribution, which can be normalized, if one takes into account the physical meaning of the parameters $x=\left(x_{1}, x_{2}, \ldots, x_{N}\right)$. One can derive the expression of the density operator $\hat{\rho}$ in terms of the probabilities and the quantizer operator $\hat{D}(x)$; it reads

$$
\hat{\rho}=\int P(x) \hat{D}(x) d x .
$$

In our present study, we concentrate on the case of discrete variables for three- and four-level atoms (qutrits and ququarts). We start with the consideration of the general properties of qudit states and construct particular cases of the density-matrix probability representation of the qudit states. There exists an infinite number of the probability representations, in view of the infinite number of different kinds of dequantizers $\hat{U}(x)$. In fact, the properties of $\hat{U}(x)$ and $\hat{D}(x)$ mean that the $d \times d$-matrices $|U(x)\rangle$ form the basis in the $d^{2}$-dimensional linear space. The vectors have the components following the 
rule of an invertible map [43] of matrices onto the vectors shown on the example of $d=2$; namely we map a given $2 \times 2$-matrix $M=\left(\begin{array}{ll}m_{11} & m_{12} \\ m_{21} & m_{22}\end{array}\right)$ onto the vector

$$
|M\rangle=\left(\begin{array}{l}
m_{11} \\
m_{12} \\
m_{21} \\
m_{22}
\end{array}\right) .
$$

In the space of four vectors for a given basis, there is an infinite number of basis vectors obtained as a linear combination of given basis vectors satisfying the completeness relation.

If the basis vector components are the probabilities, any convex sum of these vectors also determines the probabilities. If the convex sum of the initial basis vectors determines a complete set of the other basis vectors with the probability components, this means that there exist different possibilities to construct the probability representation of the state density matrices. Different kinds of such probability representations of qudit density matrices were considered in $[15,25,44]$.

In the next Sections 3 and 4 , we explicitly construct specific quantizer and dequantizer operators for the qudit states.

\section{Qudits and Their Quantizers and Dequantizers}

Here, we briefly review the properties and applications of quantizers and dequantizers for systems of qudits. Assume that we have a quantum state described by the density operator $\hat{\rho}$, for which the matrix elements of a $d$-dimensional density matrix are

$$
\rho^{j k}=\operatorname{Tr}(\hat{\rho}|k\rangle\langle j|), \quad j, k,=1, \ldots, d .
$$

Here, the vectors $|k\rangle$ are basis vectors in the $d$-dimensional Hilbert space $\mathcal{H}$ satisfying the orthogonality and normalization conditions $\langle k \mid j\rangle=\delta_{k j}$.

The matrix $\rho^{j k}$ is described by $\left(d^{2}-1\right)$ real parameters and can be mapped onto $d^{2}$ dequantizers $\hat{U}^{(n)} ; n=1, \ldots, d^{2}$. A set of the dequantizers can be selected in different ways - the choice is determined by the values we prefer to use as the symbol components. Let us choose the real and imaginary parts of matrix elements $\rho^{j k}$,

$$
\rho^{j k}=\rho_{r}^{j k}+i \rho_{c}^{j k}, \quad j<k ; \quad j, k=1, \ldots, d .
$$

In this case, one can check that the corresponding set of dequantizers is expressed in terms of operators $\hat{E}_{j k}=|k\rangle\langle j|$ as follows:

$$
\begin{aligned}
& \hat{U}_{S}^{(j j)}=\hat{E}_{j j}, \quad j=1, \ldots, d, \quad \hat{E}_{j k}=|k\rangle\langle j|, \\
& \hat{U}_{S}^{(j k)}=\frac{1}{2}\left(\hat{E}_{j k}+\hat{E}_{k j}\right), \quad \hat{U}_{c S}^{(j k)}=\frac{i}{2}\left(-\hat{E}_{j k}+\hat{E}_{k j}\right), \quad j<k ; \quad j, k=1, \ldots, d .
\end{aligned}
$$

The symbol components of the density matrix (8) corresponding to dequantizers (10) read

$$
\begin{aligned}
& \operatorname{Tr}\left(\hat{U}_{S}^{(j j)} \hat{\rho}\right)=\rho^{j j}, \quad j=1, \ldots, d \\
& \operatorname{Tr}\left(\hat{U}_{S}^{(j k)} \hat{\rho}\right)=\rho_{r}^{j k}, \quad \operatorname{Tr}\left(\hat{U}_{c S}^{(j k)} \hat{\rho}\right)=\rho_{c}^{j k}, \quad j<k ; \quad j, k=1, \ldots, d .
\end{aligned}
$$

In view of direct verification, it is easy to prove that dequantizers (10) form an orthogonal system, and the following relations are valid:

$$
\operatorname{Tr}\left(\hat{U}_{S}^{(j k)} \hat{U}_{S}^{(m n)}\right)=\frac{1}{2} \delta_{j m} \delta_{k n}, \quad \operatorname{Tr}\left(\hat{U}_{S}^{(j k)} \hat{U}_{c S}^{(m n)}\right)=0, \quad \operatorname{Tr}\left(\hat{U}_{c S}^{(j k)} \hat{U}_{c S}^{(m n)}\right)=\frac{1}{2} \delta_{j m} \delta_{k n} .
$$


From (12) immediately follows that quantizers corresponding to dequantizers (10) have the form

$$
\begin{aligned}
& \hat{D}_{S}^{(j j)}=\hat{E}_{j j}, \quad j=1, \ldots, d, \\
& \hat{D}_{S}^{(j k)}=\left(\hat{E}_{j k}+\hat{E}_{k j}\right), \quad \hat{D}_{c S}^{(j k)}=i\left(-\hat{E}_{j k}+\hat{E}_{k j}\right), \quad j<k ; \quad j, k=1, \ldots, d ;
\end{aligned}
$$

this expression for the quantizer system can be obtained in the other way. For this, we use the representation of the density matrix through its symbol components and quantizers

$$
\hat{\rho}=\sum_{j=1}^{N} K^{j} \hat{D}^{(j)}
$$

The coefficients $K^{j}$ are included in the structure of the density matrix and, choosing the matrix multipliers attributed to them, we immediately determine the type of the corresponding quantizer. Thus, in our approach, the sequence of necessary actions is as follows.

Symbol components (11) of the density operator (8) constructed, using dequantizers (10), completely define this operator. In turn, it can be restored using the numerical values. To do this, one can use the set of quantizer operators $\hat{D}^{(m n)}$ corresponding to the set of dequantizer operators (10). Then, in view of quantizers $\hat{D}^{(m n)}$ and symbol components (11), the density operator (8) can be represented as

$$
\hat{\rho}=\sum_{j=1}^{d} \rho^{j j} \hat{E}_{j j}+\sum_{j<k=1}^{d} \rho_{r}^{j k}\left(\hat{E}_{j k}+\hat{E}_{k j}\right)+\sum_{j<k=1}^{d} i \rho_{c}^{j k}\left(\hat{E}_{j k}-\hat{E}_{k j}\right) .
$$

We considered the simplest example of quantizers and dequantizers associated with the density operator (8). They are defined by (10) and (13), and corresponding symbol components of the density operator (8) and have the meaning of real and imaginary parts of matrix elements of (8). These values can be interpreted in different ways-physical, geometric, and so on. However, one can also be interested in other quantities that are connected with elements $\rho^{j k}$ by linear relations. These values $r^{\alpha \beta}$ also completely define the quantum state, and their total number is $d^{2}$. Our goal is to find a set of quantizers and dequantizers corresponding to these values, i.e., to represent them also as symbol components of the density operator.

We consider two possible cases.

(1) If we know how the original parameters $\rho^{j k}$ are expressed in terms of new parameters $r^{\alpha \beta}$,

$$
\rho^{j k}=\sum_{\alpha, \beta=1}^{d} K_{\alpha \beta}^{j k} r^{\alpha \beta}, \quad j, k, \alpha, \beta=1, \ldots, d,
$$

we substitute this expression in the density operator (8) and immediately arrive at the expression for $d^{2}$ quantizers $\hat{D}^{(\alpha \beta)}$. This means that the density operator (8) can be written in terms of parameters $r^{\alpha \beta}$ as follows:

$$
\hat{\rho}=\sum_{\alpha, \beta=1}^{d} r^{\alpha \beta} \hat{D}^{(\alpha \beta)}
$$

In fact, the representation of the density operator (8) as (17) is the definition of quantizers $\hat{D}^{(\alpha \beta)}$; to find them, one does not need to perform any calculations but just substitute (16) into (8). In order to find an explicit form of dequantizers, we need to consider relations (16) as a system of linear equations for unknown quantities $r^{\alpha \beta}$. Solving this system, we obtain the expression for the new parameters $r^{\alpha \beta}$ through the matrix elements $\rho^{j k}$, 


$$
r^{\alpha \beta}=\sum_{j k=1}^{d} L_{j k}^{\alpha \beta} \rho^{j k}, \quad j, k, \alpha, \beta=1, \ldots, d ;
$$

this relationship allows one to find a dequantizer, for which the value $r^{\alpha \beta}$ is a symbol component of the density operator (8). This dequantizer is a linear combination of dequantizer operators (13) that follows from a linear combination (18) for matrices of dequantizer operators,

$$
\hat{U}^{\alpha \beta}=\sum_{j k=1}^{d} L_{j k}^{\alpha \beta} \hat{U}_{S}^{(j k)}, \quad j, k, \alpha, \beta=1, \ldots, d .
$$

(2) The second possibility consists of the fact that we know how the new parameters $r^{\alpha \beta}$ are expressed in terms of matrix elements $\rho^{j k}$. In other words, we assume that (18) is valid from the very beginning. Then, in view of this relation, we can immediately write expression (19) for dequantizers $\hat{U}^{\alpha \beta}$. Thus, in this case, expression (19) for dequantizer operators arises automatically from (18), which expresses the new parameters through the original ones.

To find an explicit form of quantizers corresponding to dequantizer operators (19), we need to consider (18) as a system of linear equations for unknown quantities $\rho^{j k}$, i.e., to perform the inverse operation with respect to the one we did in the previous case (1). Solving the system of equations (18) we arrive at a formula similar to (16), which expresses the matrix elements $\rho^{j k}$ through the new parameters $r^{\alpha \beta}$. Now, exactly as in the previous case (1), one must substitute these expressions in matrix (8) and select the numerical parameters $r^{\alpha \beta}$. The density matrix (8) will take the form (17), where $\hat{D}^{(\alpha \beta)}$ are quantizers corresponding to dequantizer matrices (19).

Thus, we obtained that any sets of quantizers and dequantizers can be expressed in terms of the original sets of operators (10). In this case, depending on how the new parameters are related to the matrix elements of the density matrix, there exist two possibilities:

(i) If the matrix elements $\rho^{j k}$ are expressed in terms of the new parameters $r^{\alpha \beta}$ using relations (16), the quantizers can be obtained directly using this relation and, to find the dequantizer matrices, one needs to solve the system of equations (16).

(ii) If relation (18) is initially available, we first use it to construct dequantizer matrices and then, solving this system of equations, we obtain the opportunity to construct the corresponding set of quantizers.

In both cases, one must solve the system of equations that connects the matrix elements of the density matrix with a new set of parameters. In this respect, our approach is equivalent to the standard method for constructing a set of quantizers by dequantizers, which is based on employing the orthogonality relation. In both cases, there is a system of $d^{2}$ linear equations for $d^{2}$ unknowns. However, there is an important difference between the two approaches. The standard approach uses only an explicit form of dequantizer operators, and relations (16) and (18) are not taken into account. But these systems can be much simpler than the systems based on the use of the orthogonality relation, as is often the case in practice. Therefore, the method of constructing quantizer-dequantizer systems proposed here is much more reasonable.

\section{Example of the Qudit State}

In our previous paper [21], we considered the qutrit-state density operator $\hat{\rho}_{3}$. This qutrit-state density operator was studied in detail in [45,46]. In [27], we constructed several sets of quantizer and dequantizer operators for the density operator $\hat{\rho}_{3}$, applied them to study the density operator $\hat{\rho}_{3}$, and analyze the properties of quantizers and dequantizers. Below we consider the general case of an arbitrary qudit-state density operator. The aim of this consideration is to map the density operator of an arbitrary qudit state onto the probability distributions identified with the state. We use the qutrit and ququart states 
as examples of the probability representation of quantum states. A formalism to develop such a probability representation is the formalism of quantizer and dequantizer operators, which we present for qutrit and ququart systems to be studied below on the example of the physical phenomenon called the parametric down-conversion.

In Sections 4-6, we consider the matrix representation of the density operator and quantizer-dequantizer operators, i.e., instead of the operator notation $\hat{\rho}, \hat{\mathcal{D}}$, and $\hat{\mathcal{U}}$, we use the notation $\rho, D$, and $U$ for their matrices-the operator $\hat{E}_{j k}$ is given by the matrix $E_{j k}$, and this matrix includes one nonzero element, which is equal to unity and located at the intersection of the $j$ th row and the $k$ th column.

In a generic case, the density matrix of a qudit state reads

$$
\rho=\left\|\rho_{j k}\right\|, \quad j, k=1, \ldots, d .
$$

We consider the case where the matrix elements $\rho_{j k}$ are

$$
\begin{aligned}
& \rho_{11}=\sum_{j=2}^{d}(d-1) p_{3}^{(j j)}-d+2, \quad \rho_{j j}=1-(d-1) p_{3}^{(j j)}, \quad j=2, \ldots, d, \\
& \rho_{j k}=\frac{1}{2}\left(d p_{1}^{(j k)}-1\right)-\frac{i}{2}\left(d p_{2}^{(j k)}-1\right), \quad j<k ; \quad j, k=1, \ldots, d .
\end{aligned}
$$

In the previous section, we developed a generic scheme for constructing sets of quantizer-dequantizer operators and analyzed their relations. Now we show how this scheme is implemented to the qudit state; for this, we consider two sets of dequantizers.

The components of symbol of the density matrix (20) corresponding to dequantizers (10) read

$$
\begin{aligned}
& \operatorname{Tr}\left(\hat{U}_{S}^{(11)} \hat{\rho}\right)=m^{11}=\sum_{j=2}^{d}(d-1) p_{3}^{(j j)}-d+2, \\
& \operatorname{Tr}\left(\hat{U}_{S}^{(j j)} \hat{\rho}\right)=m^{j j}=1-(d-1) p_{3}^{(j j)}, \quad j=2, \ldots, d, \\
& \operatorname{Tr}\left(\hat{U}_{S}^{(j k)} \hat{\rho}\right)=m^{j k}=d p_{1}^{(j k)}-1 / 2, \\
& \operatorname{Tr}\left(\hat{U}_{c S}^{(j k)} \hat{\rho}\right)=m_{c}^{j k}=d p_{2}^{(j k)}-1 / 2, \quad j<k ; \quad j, k=1, \ldots, d .
\end{aligned}
$$

Then the set of quantizers corresponding to the dequantizers matches exactly to the normalizing coefficients with the set of dequantizers (10) given in matrix form

$$
\begin{aligned}
& D_{S}^{(j j)}=E_{j j}, \quad j=1, \ldots, d, \\
& D_{S}^{(j k)}=\left(E_{j k}+E_{k j}\right), \quad D_{c S}^{(j k)}=i\left(-E_{j k}+E_{k j}\right), \quad j<k ; \quad j, k=1, \ldots, d .
\end{aligned}
$$

We see that dequantizers (10) along with quantizers (23) form the self-dual set.

The density operator of the qudit state can be presented in the form

$$
\begin{aligned}
\hat{\rho}= & \hat{D}_{S}^{(11)}\left(\sum_{j=2}^{d}(d-1) p_{3}^{(j j)}-d+2\right)+\sum_{j=2}^{d} \hat{D}_{S}^{(j j)}\left(1-(d-1) p_{3}^{(j j)}\right) \\
& +\sum_{j<k} \hat{D}_{S}^{(j k)}\left(d p_{1}^{(j k)}-1 / 2\right)+\sum_{j<k} \hat{D}_{S c}^{(j k)}\left(d p_{2}^{(j k)}-1 / 2\right) .
\end{aligned}
$$

One can see that, in this representation, quantizers (23) are coefficients of the components of symbol of the density matrix. 
The second set of dequantizers is given by matrices

$$
\begin{aligned}
& U^{(11)}=\frac{I}{d}, U^{(j j)}=\frac{1}{d-1}\left(I-E_{j j}\right), j=2, \ldots, d, \\
& U^{(j k)}=\frac{1}{d}\left(I+E_{j k}+E_{k j}\right), U_{c}^{(j k)}=\frac{1}{d}\left(I+i\left(-E_{j k}+E_{k j}\right)\right), j<k ; j, k=1, \ldots, d,
\end{aligned}
$$

where $I$ is a $d \times d$ identity matrix. These dequantizers have traces equal to one, and they are Hermitian positive definite matrices; therefore, they can be considered as density matrices for some physical states, and they are not projectors.

The corresponding set of quantizers includes operators acting on the $d$-dimensional Hilbert space; these operators have the matrices

$$
\begin{array}{r}
D^{(11)}=d I+(-d+1) E_{11}+\frac{1}{2}\left((-1+i) \sum_{m<n} E_{m n}+(-1-i) \sum_{m<n} E_{n m}\right), \\
D^{(j j)}=(d-1)\left(E_{11}-E_{j j}\right), \quad j=2, \ldots, d . \\
D^{(j k)=\frac{1}{2} d\left(E_{j k}+E_{k j}\right), \quad D_{c}^{(j k)}=} \frac{i}{2} d\left(-E_{j k}+E_{k j}\right), \quad j<k ; \quad j, k=1, \ldots, d .
\end{array}
$$

The matrices (25) and (26) satisfy the orthogonality condition

$$
\operatorname{Tr}\left(U^{(j k)} D^{(m n)}\right)=\delta(j m) \delta(k n) .
$$

The components of symbol of the density matrix (21) corresponding to dequantizers (25) are the probabilities $p_{l}^{(j k)} ; l=1,2,3$ and $j, k=1, \ldots, d$; they read

$$
\begin{array}{lll}
\operatorname{Tr}\left(U^{(11)} \rho\right)=p_{3}^{(11)}=\frac{1}{d^{\prime}} & \operatorname{Tr}\left(U^{(j j)} \rho\right)=p_{3}^{(j j)}, & j=2, \ldots, d, \\
\operatorname{Tr}\left(U^{(j k)} \rho\right)=p_{1}^{(j k)}, & \operatorname{Tr}\left(U_{c}^{(j k)} \rho\right)=p_{2}^{(j k)}, & j<k ; j, k=1, \ldots, d .
\end{array}
$$

With the set of quantizers (22) and symbol (24), one can reconstruct the density matrix (20),

$$
\rho=\sum_{j=1}^{d} D^{(j j)} p_{3}^{(j j)}+\sum_{j<k} D^{(j k)} p_{1}^{(j k)}+\sum_{j<k} D_{c}^{(j k)} p_{2}^{(j k)} .
$$

As mentioned above the usual method to construct the set of quantizers is to use the orthogonality condition (27), i.e., this method assumes to consider (27) as a system of algebraic equations. The solutions to this system of equations are matrix elements of operators $\hat{D}^{(m n)}$, which form the set of quantizers. But this way may be too tedious. However, in order to find a set of quantizers corresponding to a certain set of dequntaizers, it is not necessary to solve (27), but it is enough to calculate components (28) of symbol of the density operator and present it in the form (29). Then one has to compare the original matrix form of the density matrix with its representation (29), and this comparison immediately provides the explicit form of quantizers.

This approach is valid not only for the density operator but it can be used for the other operators as well. Now let us look how sets of quantizers and dequantizers, whose symbol components are connected by linear relation (18), are related to each other; in the general case, this relation is given by (19). Now we show how this dependence looks for qudit states (21). For such a state, we consider two sets of dequantizers (10) and (25) with the corresponding sets of symbol components (22) and (28), and relations (18) take the form 


$$
\begin{array}{llrl}
p_{3}^{(11)}=\sum_{l=1}^{d} m^{l l}=1, & p_{3}^{(j j)}=\sum_{l=1}^{d} m^{l l}-m^{j j}=1-m^{j j}, & j=2, \ldots, d, \\
p_{1}^{(j k)}=m_{1}^{j k}+\frac{1}{2} \sum_{l=1}^{d} m^{l l}, & p_{2}^{(j k)}=m_{2}^{j k}+\frac{1}{2} \sum_{l=1}^{d} m^{l l}, & j<k ; j, k=1, \ldots, d .
\end{array}
$$

We see that this system is not tedious. In view of equation (29), one can find the dequantizers corresponding to symbol components (28).

\section{Example of the Ququart State}

As an example, we consider the case $d=4$. This example corresponds to the ququart state; this state can be either pure or mixed one. The states are described by the Hermitian density operator $\hat{\rho}$ acting on the four-dimensional Hilbert space. The density operator is associated with the density matrix, which we express in terms of probabilities. We will consider the case of photon states created in the down-conversion process.

We write the density $4 \times 4$-matrix of the ququart state in terms of four columns denoted as

$$
\rho=\left(\begin{array}{llll}
A & B & C & D
\end{array}\right),
$$

with

$$
\begin{aligned}
& 3 p_{3}^{(22)}+3 p_{3}^{(33)}+3 p_{3}^{(44)}-2 \\
& A=\left(2 p_{1}^{(12)}-1 / 2\right)+i\left(2 p_{2}^{(12)}-1 / 2\right) \\
& \left(2 p_{1}^{(13)}-1 / 2\right)+i\left(2 p_{2}^{(13)}-1 / 2\right) \\
& \left(2 p_{1}^{(14)}-1 / 2\right)+i\left(2 p_{2}^{(14)}-1 / 2\right) \\
& B= \\
& \left(2 p_{1}^{(12)}-1 / 2\right)-i\left(2 p_{2}^{(12)}-1 / 2\right) \\
& 1-3 p_{3}^{(22)} \\
& \left(2 p_{1}^{(23)}-1 / 2\right)+i\left(2 p_{2}^{(23)}-1 / 2\right) \\
& \left(2 p_{1}^{(13)}-1 / 2\right)-i\left(2 p_{2}^{(13)}-1 / 2\right) \\
& C=\begin{array}{c}
\left(2 p_{1}^{(23)}-1 / 2\right)-i\left(2 p_{2}^{(23)}-1 / 2\right) \\
1-3 p_{3}^{(33)} \\
\left(2 p_{1}^{(34)}-1 / 2\right)+i\left(2 p_{2}^{(34)}-1 / 2\right)
\end{array}, \quad D=\begin{array}{c}
\left(2 p_{1}^{(24)}-1 / 2\right)-i\left(2 p_{2}^{(24)}-1 / 2\right) \\
\left(2 p_{1}^{(34)}-1 / 2\right)-i\left(2 p_{2}^{(34)}-1 / 2\right) \\
1-3 p_{3}^{(44)}
\end{array} . \\
& \left(2 p_{1}^{(24)}-1 / 2\right)+i\left(2 p_{2}^{(24)}-1 / 2\right) \\
& \left(2 p_{1}^{(14)}-1 / 2\right)-i\left(2 p_{2}^{(14)}-1 / 2\right)
\end{aligned}
$$

Now the matrices of dequantizers (25) take the form

$$
\begin{aligned}
& U^{(11)}=\frac{1}{4}\left(\begin{array}{cccc}
1 & 0 & 0 & 0 \\
0 & 1 & 0 & 0 \\
0 & 0 & 1 & 0 \\
0 & 0 & 0 & 1
\end{array}\right), \quad U^{(22)}=\frac{1}{3}\left(\begin{array}{cccc}
1 & 0 & 0 & 0 \\
0 & 0 & 0 & 0 \\
0 & 0 & 1 & 0 \\
0 & 0 & 0 & 1
\end{array}\right), \quad U^{(33)}=\frac{1}{3}\left(\begin{array}{cccc}
1 & 0 & 0 & 0 \\
0 & 1 & 0 & 0 \\
0 & 0 & 0 & 0 \\
0 & 0 & 0 & 1
\end{array}\right), \\
& U^{(44)}=\frac{1}{3}\left(\begin{array}{llll}
1 & 0 & 0 & 0 \\
0 & 1 & 0 & 0 \\
0 & 0 & 1 & 0 \\
0 & 0 & 0 & 0
\end{array}\right), \quad U^{(12)}=\frac{1}{4}\left(\begin{array}{cccc}
1 & 1 & 0 & 0 \\
1 & 1 & 0 & 0 \\
0 & 0 & 1 & 0 \\
0 & 0 & 0 & 1
\end{array}\right), \quad U_{c}^{(12)}=\frac{1}{4}\left(\begin{array}{cccc}
1 & -i & 0 & 0 \\
i & 1 & 0 & 0 \\
0 & 0 & 1 & 0 \\
0 & 0 & 0 & 1
\end{array}\right) \text {, } \\
& U^{(13)}=\frac{1}{4}\left(\begin{array}{llll}
1 & 0 & 1 & 0 \\
0 & 1 & 0 & 0 \\
1 & 0 & 1 & 0 \\
0 & 0 & 0 & 1
\end{array}\right), \quad U_{c}^{(13)}=\frac{1}{4}\left(\begin{array}{cccc}
1 & 0 & -i & 0 \\
0 & 1 & 0 & 0 \\
i & 0 & 1 & 0 \\
0 & 0 & 0 & 1
\end{array}\right), \quad U^{(14)}=\frac{1}{4}\left(\begin{array}{cccc}
1 & 0 & 0 & 1 \\
0 & 1 & 0 & 0 \\
0 & 0 & 1 & 0 \\
1 & 0 & 0 & 1
\end{array}\right) \text {, } \\
& U^{(23)}=\frac{1}{4}\left(\begin{array}{llll}
1 & 0 & 0 & 0 \\
0 & 1 & 1 & 0 \\
0 & 1 & 1 & 0 \\
0 & 0 & 0 & 1
\end{array}\right), \quad U_{c}^{(23)}=\frac{1}{4}\left(\begin{array}{cccc}
1 & 0 & 0 & 0 \\
0 & 1 & -i & 0 \\
0 & i & 1 & 0 \\
0 & 0 & 0 & 1
\end{array}\right), \quad U^{(24)}=\frac{1}{4}\left(\begin{array}{cccc}
1 & 0 & 0 & 0 \\
0 & 1 & 0 & 1 \\
0 & 0 & 1 & 0 \\
0 & 1 & 0 & 1
\end{array}\right) \text {, }
\end{aligned}
$$




$$
\begin{aligned}
U_{c}^{(24)}=\frac{1}{4}\left(\begin{array}{cccc}
1 & 0 & 0 & 0 \\
0 & 1 & 0 & -i \\
0 & 0 & 1 & 0 \\
0 & i & 0 & 1
\end{array}\right), U^{(34)}=\frac{1}{4}\left(\begin{array}{cccc}
1 & 0 & 0 & 0 \\
0 & 1 & 0 & 0 \\
0 & 0 & 1 & 1 \\
0 & 0 & 1 & 1
\end{array}\right), U_{c}^{(34)}=\frac{1}{4}\left(\begin{array}{cccc}
1 & 0 & 0 & 0 \\
0 & 1 & 0 & 0 \\
0 & 0 & 1 & -i \\
0 & 0 & i & 1
\end{array}\right), \\
U_{c}^{(14)}=\frac{1}{4}\left(\begin{array}{cccc}
1 & 0 & 0 & -i \\
0 & 1 & 0 & 0 \\
0 & 0 & 1 & 0 \\
i & 0 & 0 & 1
\end{array}\right) .
\end{aligned}
$$

Dequantizers (32) have traces equal to one, also they are Hermitian positive definite matrices; therefore, we can consider them as density matrices for some physical states.

The components of symbol of the density matrix (31) corresponding to dequantizers (32) read

$$
\begin{array}{llll}
\operatorname{Tr}\left(U^{(11)} \rho\right)=p_{3}^{(11)}=1 / 4, & \operatorname{Tr}\left(U^{22} \rho\right)=p_{3}^{(22)}, & \operatorname{Tr}\left(U^{33} \rho\right)=p_{3}^{(33)}, & \operatorname{Tr}\left(U^{44} \rho\right)=p_{3}^{(44),}, \\
\operatorname{Tr}\left(U^{(12)} \rho\right)=p_{1}^{(12)}, & \operatorname{Tr}\left(U^{(13)} \rho\right)=p_{1}^{(13)}, & \operatorname{Tr}\left(U^{(14)} \rho\right)=p_{1}^{(14)}, & \operatorname{Tr}\left(U^{(23)} \rho\right)=p_{1}^{(23),}, \\
\operatorname{Tr}\left(U^{(24)} \rho\right)=p_{1}^{(24)}, & \operatorname{Tr}\left(U^{(34)} \rho\right)=p_{1}^{(34)}, & \operatorname{Tr}\left(U_{c}^{(12)} \rho\right)=p_{2}^{(12)}, & \operatorname{Tr}\left(U_{c}^{(13)} \rho\right)=p_{2}^{(13),}, \\
\operatorname{Tr}\left(U_{c}^{(14)} \rho\right)=p_{2}^{(14)}, & \operatorname{Tr}\left(U_{c}^{(23)} \rho\right)=p_{2}^{(23)}, & \operatorname{Tr}\left(U_{c}^{(24)} \rho\right)=p_{2}^{(24)}, & \operatorname{Tr}\left(U_{c}^{(34)} \rho\right)=p_{2}^{(34)} .
\end{array}
$$

The set of quantizers corresponding to dequantizers (32) are

$$
\begin{aligned}
& D^{(11)}=2\left(\begin{array}{cccc}
-4 & -1+i & -1+i & -1+i \\
-1-i & 2 & -1+i & -1+i \\
-1-i & -1-i & 2 & -1+i \\
-1-i & -1-i & -1-i & 2
\end{array}\right), \quad D^{(22)}=\left(\begin{array}{cccc}
3 & 0 & 0 & 0 \\
0 & -3 & 0 & 0 \\
0 & 0 & 0 & 0 \\
0 & 0 & 0 & 0
\end{array}\right) \\
& D^{(33)}=\left(\begin{array}{cccc}
3 & 0 & 0 & 0 \\
0 & 0 & 0 & 0 \\
0 & 0 & -3 & 0 \\
0 & 0 & 0 & 0
\end{array}\right), \quad D^{(12)}=\left(\begin{array}{cccc}
0 & 2 & 0 & 0 \\
2 & 0 & 0 & 0 \\
0 & 0 & 0 & 0 \\
0 & 0 & 0 & 0
\end{array}\right), \quad D_{c}^{(12)}=\left(\begin{array}{cccc}
0 & -2 i & 0 & 0 \\
2 i & 0 & 0 & 0 \\
0 & 0 & 0 & 0 \\
0 & 0 & 0 & 0
\end{array}\right) \text {, } \\
& D^{(13)}=\left(\begin{array}{cccc}
0 & 0 & 2 & 0 \\
0 & 0 & 0 & 0 \\
2 & 0 & 0 & 0 \\
0 & 0 & 0 & 0
\end{array}\right), \quad D_{c}^{(13)}=\left(\begin{array}{cccc}
0 & 0 & -2 i & 0 \\
0 & 0 & 0 & 0 \\
2 i & 0 & 0 & 0 \\
0 & 0 & 0 & 0
\end{array}\right), \quad D^{(14)}=\left(\begin{array}{cccc}
0 & 0 & 0 & 2 \\
0 & 0 & 0 & 0 \\
0 & 0 & 0 & 0 \\
2 & 0 & 0 & 0
\end{array}\right) \text {, } \\
& D^{(23)}=\left(\begin{array}{cccc}
0 & 0 & 0 & 0 \\
0 & 0 & 2 & 0 \\
0 & 2 & 0 & 0 \\
0 & 0 & 0 & 0
\end{array}\right), \quad D_{c}^{(23)}=\left(\begin{array}{cccc}
0 & 0 & 0 & 0 \\
0 & 0 & -2 i & 0 \\
0 & 2 i & 0 & 0 \\
0 & 0 & 0 & 0
\end{array}\right), \quad D^{(24)}=\left(\begin{array}{llll}
0 & 0 & 0 & 0 \\
0 & 0 & 0 & 2 \\
0 & 0 & 0 & 0 \\
0 & 2 & 0 & 0
\end{array}\right) \text {, } \\
& D_{c}^{(24)}=\left(\begin{array}{cccc}
0 & 0 & 0 & 0 \\
0 & 0 & 0 & -2 i \\
0 & 0 & 0 & 0 \\
0 & 2 i & 0 & 0
\end{array}\right), \quad D^{(34)}=\left(\begin{array}{cccc}
0 & 0 & 0 & 0 \\
0 & 0 & 0 & 0 \\
0 & 0 & 0 & 2 \\
0 & 0 & 2 & 0
\end{array}\right), \quad D_{c}^{(34)}=\left(\begin{array}{cccc}
0 & 0 & 0 & 0 \\
0 & 0 & 0 & 0 \\
0 & 0 & 0 & -2 i \\
0 & 0 & 2 i & 0
\end{array}\right) \text {, } \\
& D^{(44)}=\left(\begin{array}{cccc}
3 & 0 & 0 & 0 \\
0 & 0 & 0 & 0 \\
0 & 0 & 0 & 0 \\
0 & 0 & 0 & -3
\end{array}\right), \quad D_{c}^{(14)}=\left(\begin{array}{cccc}
0 & 0 & 0 & -2 i \\
0 & 0 & 0 & 0 \\
0 & 0 & 0 & 0 \\
2 i & 0 & 0 & 0
\end{array}\right) \text {. }
\end{aligned}
$$

In view of quantizers (34) and symbol (33), the density matrix (31) can be presented in the form

$$
\rho=D^{(11)} p_{3}^{(11)}+D^{(22)} p_{3}^{(22)}+D^{(33)} p_{3}^{(33)}+D^{(44)} p_{3}^{(44)}+D^{(12)} p_{1}^{(12)}+D^{(13)} p_{1}^{(13)}
$$




$$
\begin{aligned}
& +D^{(14)} p_{1}^{(14)}+D^{(23)} p_{1}^{(23)}+D^{(24)} p_{1}^{(24)}+D^{(34)} p_{1}^{(34)}+D_{c}^{(12)} p_{2}^{(12)}+D_{c}^{(13)} p_{2}^{(13)} \\
& +D_{c}^{(14)} p_{2}^{(14)}+D_{c}^{(23)} p_{2}^{(23)}+D_{c}^{(24)} p_{2}^{(24)}+D_{c}^{(34)} p_{2}^{(34)}
\end{aligned}
$$

The other set of dequantizer matrices is given by operators (10); in the case of ququart, they read

$$
\begin{aligned}
& U_{S}^{(11)}=\left(\begin{array}{cccc}
1 & 0 & 0 & 0 \\
0 & 0 & 0 & 0 \\
0 & 0 & 0 & 0 \\
0 & 0 & 0 & 0
\end{array}\right), \quad U_{S}^{(22)}=\left(\begin{array}{cccc}
0 & 0 & 0 & 0 \\
0 & 1 & 0 & 0 \\
0 & 0 & 0 & 0 \\
0 & 0 & 0 & 0
\end{array}\right), \quad U_{S}^{(33)}=\left(\begin{array}{cccc}
0 & 0 & 0 & 0 \\
0 & 0 & 0 & 0 \\
0 & 0 & 1 & 0 \\
0 & 0 & 0 & 0
\end{array}\right) \\
& U_{S}^{(44)}=\left(\begin{array}{llll}
0 & 0 & 0 & 0 \\
0 & 0 & 0 & 0 \\
0 & 0 & 0 & 0 \\
0 & 0 & 0 & 1
\end{array}\right), \quad U_{S}^{(12)}=\frac{1}{2}\left(\begin{array}{cccc}
0 & 1 & 0 & 0 \\
1 & 0 & 0 & 0 \\
0 & 0 & 0 & 0 \\
0 & 0 & 0 & 0
\end{array}\right), \quad U_{S c}^{(12)}=\frac{1}{2}\left(\begin{array}{cccc}
0 & -i & 0 & 0 \\
i & 0 & 0 & 0 \\
0 & 0 & 0 & 0 \\
0 & 0 & 0 & 0
\end{array}\right) \text {, } \\
& U_{S}^{(13)}=\frac{1}{2}\left(\begin{array}{llll}
0 & 0 & 1 & 0 \\
0 & 0 & 0 & 0 \\
1 & 0 & 0 & 0 \\
0 & 0 & 0 & 0
\end{array}\right), \quad U_{S c}^{(13)}=\frac{1}{2}\left(\begin{array}{cccc}
0 & 0 & -i & 0 \\
0 & 0 & 0 & 0 \\
i & 0 & 0 & 0 \\
0 & 0 & 0 & 0
\end{array}\right), \quad U_{S}^{(14)}=\frac{1}{2}\left(\begin{array}{cccc}
0 & 0 & 0 & 1 \\
0 & 0 & 0 & 0 \\
0 & 0 & 0 & 0 \\
1 & 0 & 0 & 0
\end{array}\right) \text {, } \\
& U_{S}^{(23)}=\frac{1}{2}\left(\begin{array}{cccc}
0 & 0 & 0 & 0 \\
0 & 0 & 1 & 0 \\
0 & 1 & 0 & 0 \\
0 & 0 & 0 & 0
\end{array}\right), \quad U_{S c}^{(23)}=\frac{1}{2}\left(\begin{array}{cccc}
0 & 0 & 0 & 0 \\
0 & 0 & -i & 0 \\
0 & i & 0 & 0 \\
0 & 0 & 0 & 0
\end{array}\right), \quad U_{S}^{(24)}=\frac{1}{2}\left(\begin{array}{cccc}
0 & 0 & 0 & 0 \\
0 & 0 & 0 & 1 \\
0 & 0 & 0 & 0 \\
0 & 1 & 0 & 0
\end{array}\right) \text {, } \\
& U_{S c}^{(24)}=\frac{1}{2}\left(\begin{array}{cccc}
0 & 0 & 0 & 0 \\
0 & 0 & 0 & -i \\
0 & 0 & 0 & 0 \\
0 & i & 0 & 0
\end{array}\right), \quad U_{S}^{(34)}=\frac{1}{2}\left(\begin{array}{cccc}
0 & 0 & 0 & 0 \\
0 & 0 & 0 & 0 \\
0 & 0 & 0 & 1 \\
0 & 0 & 1 & 0
\end{array}\right), \quad U_{S c}^{(34)}=\frac{1}{2}\left(\begin{array}{cccc}
0 & 0 & 0 & 0 \\
0 & 0 & 0 & 0 \\
0 & 0 & 0 & -i \\
0 & 0 & i & 0
\end{array}\right) \text {, } \\
& U_{S c}^{(14)}=\frac{1}{2}\left(\begin{array}{cccc}
0 & 0 & 0 & -i \\
0 & 0 & 0 & 0 \\
0 & 0 & 0 & 0 \\
i & 0 & 0 & 0
\end{array}\right)
\end{aligned}
$$

Since the set of dequantizers (36) is self-dual, all operators from this set are orthogonal to each other; therefore, the corresponding quantizers are exactly the same as dequantizers up to a factor.

\section{Qutrit and Ququart in the Spontaneous Parametric Down-Conversion Process}

The simplest states used in quantum information are qubits given as superpositions of any two basis quantum states. If a system under consideration consists of two subsystems, each being the qubit state, the bipartite wave function of the composite two-qubit state, in general case, is given by a sum of direct products of the qubit wave functions. Biphoton states can be formed using the spontaneous parametric down-conversion (SPDC) process [47]. It is a nonlinear optical process, where the pump photon spontaneously splits into two photons of lower energies. It is an important process in quantum optics for the generation of single photons and entangled photon pairs. The SPDC process has found many applications in various fields of research; see, for example, [48].

Selecting the crystal and its orientation along with the manipulation of the polarization of SPDC-generated photons, it is possible to construct the biphoton qutrit and ququart states of a general type $[35,47,49,50]$. 
In the simplest case, the SPDC Hamiltonian $\mathcal{H}$ reads

$$
\hat{\mathcal{H}}=\hbar\left(\omega_{p} \hat{b}_{p}^{\dagger} \hat{b}_{p}+\omega_{s} \hat{a}_{s}^{\dagger} \hat{a}_{s}+\omega_{i} \hat{a}_{i}^{\dagger} \hat{a}_{i}+g \hat{a}_{s}^{\dagger} \hat{a}_{i}^{\dagger} \hat{b}_{p}+g^{*} \hat{a}_{s} \hat{a}_{i} \hat{b}_{p}^{+}\right), \quad \omega_{p}=\omega_{s}+\omega_{i} .
$$

Here, $\hat{b}_{p}^{+}$and $\hat{b}_{p}$ are the creation and annihilation operators of the pump mode, the $\hat{a}_{s}^{\dagger}, \hat{a}_{s}$ and $\hat{a}_{i}^{\dagger}, \hat{a}_{i}$ are the creation and annihilation operators for the photons of the signal (s) and idle $(i)$ modes. The mathematical properties of such three-boson and four-boson Hamiltonians were investigated in [51-53].

During the SPDC process, the photon states corresponding to the horizontal $H$ and the vertical $V$ polarizations occur. Also it is possible that all photons with the same polarization have the same frequency. For example, photons with the vertical polarization have frequency $\omega_{i}$, and photons with the horizontal polarization have frequency $\omega_{s}$. In this case, there are three basis states. The state vector $\left|\Psi^{(3)}\right\rangle$ describes the photons created in the SPDC process, and this state of photons is the pure state; the state vector reads

$$
\begin{aligned}
\left|\Psi^{(3)}\right\rangle & =C_{1}\left|2_{H}, 0\right\rangle+C_{2}\left|1_{H}, 1_{V}\right\rangle+C_{3}\left|0,2_{V}\right\rangle \\
& =\frac{1}{\sqrt{2}}\left(C_{1} \hat{a}_{H}^{+2}+\sqrt{2} C_{2} \hat{a}_{H}^{\dagger} \hat{b}_{V}^{+}+C_{3} \hat{b}_{V}^{+2}\right)|0\rangle, \quad\left|C_{1}\right|^{2}+\left|C_{2}\right|^{2}+\left|C_{3}\right|^{2}=1,
\end{aligned}
$$

where $|0\rangle$ is the vacuum state and $\hat{a}_{H}^{+}, \hat{a}_{H}$ and $\hat{b}_{V}^{+}, \hat{b}_{V}$ are the creation and annihilation operators of the horizontal $H$ and vertical $V$ modes, respectively.

Now we discuss the physical meaning of the qutrit-state density matrix elements, using the probability representation of quantum states. The corresponding density matrix reads

$$
\rho_{\Psi(3)}=\left(\begin{array}{lll}
C_{1}^{1 *} C_{1}^{1} & C_{2}^{1 *} C_{1}^{1} & C_{3}^{1 *} C_{1}^{1} \\
C_{1}^{1 *} C_{2}^{1} & C_{2}^{1 *} C_{2}^{1} & C_{3}^{1 *} C_{2}^{1} \\
C_{1}^{1 *} C_{3}^{1} & C_{2}^{1 *} C_{3}^{1} & C_{3}^{1 *} C_{3}^{1}
\end{array}\right)
$$

In view of (21), after some algebra we arrive at the general form of qutrit-state density matrix written in the introduced probability representation of the qutrit state; it is

$$
\rho_{3}=\frac{1}{2}\left(\begin{array}{ccc}
4 p_{3}^{(33)}+4 p_{3}^{(22)}-2 & \left(3 p_{1}^{(12)}-1\right)-i\left(3 p_{2}^{(12)}-1\right) & \left(3 p_{1}^{(13)}-1\right)-i\left(3 p_{2}^{(13)}-1\right) \\
\left(3 p_{1}^{(12)}-1\right)+i\left(3 p_{2}^{(12)}-1\right) & 2-4 p_{3}^{(22)} & \left(3 p_{1}^{(23)}-1\right)-i\left(3 p_{2}^{(23)}-1\right) \\
\left(3 p_{1}^{(13)}-1\right)+i\left(3 p_{2}^{(13)}-1\right) & \left(3 p_{1}^{(23)}-1\right)+i\left(3 p_{2}^{(23)}-1\right) & 2-4 p_{3}^{(33)}
\end{array}\right) .
$$

It is a generic form of the density matrix of an arbitrary qutrit state, both mixed and pure ones. So there exist some relations between matrix elements of matrices (39) and (40); two of them read

$$
p_{3}^{(22)}=\frac{1}{2}\left(1-\left|C_{2}^{1}\right|^{2}\right), \quad p_{3}^{(33)}=\frac{1}{2}\left(1-\left|C_{3}^{1}\right|^{2}\right) .
$$

The real nonnegative numbers $p_{3}^{(22)}$ and $p_{3}^{(33)}$ are probabilities determined by (33) as traces of products of the density matrix and dequantizers.

Now we can explain the physical meaning of these probabilities. In quantum mechanics, each physical system state is associated with a Hilbert space. Any measurement upon a physical system is represented by a self-adjoint operator on the Hilbert space called the observable. The eigenvectors of such an operator form an orthonormal basis for the Hilbert space, and each possible outcome of the measurement corresponds to one of the vectors constituting the basis. For each measurement which can be fulfilled with the system, the probability distribution over the outcomes of the measurement can be calculated, in view of the density operator. Such a procedure is known as Born's rule [54]. Born's rule states that

$$
p\left(x_{i}\right)=\operatorname{Tr}\left(\hat{P}_{i} \hat{\rho}\right),
$$


where $\hat{\rho}$ is the density operator, and $\hat{P}_{i}$ is the projection operator onto the basis vector corresponding to the measurement outcome $x_{i}$. Born's rule associates the probability with each unit vector in the Hilbert space in such a way that the sum of these probabilities is equal to unity for any set of unit vectors constituting an orthonormal basis.

In the case of qutrit-state vector (38), the state vectors

$$
\vec{v}_{(2,0)}=\left|2_{H}, 0\right\rangle, \quad \vec{v}_{(1,1)}=\left|1_{H}, 1_{V}\right\rangle, \quad \vec{v}_{(0,2)}=\left|0,2_{V}\right\rangle
$$

form the basis in the three-dimensional Hilbert space. The corresponding matrices of the projectors read

$$
\hat{P}_{(2,0)}=\left(\begin{array}{ccc}
1 & 0 & 0 \\
0 & 0 & 0 \\
0 & 0 & 0
\end{array}\right), \quad \hat{P}_{(1,1)}=\left(\begin{array}{ccc}
0 & 0 & 0 \\
0 & 1 & 0 \\
0 & 0 & 0
\end{array}\right), \quad \hat{P}_{(0,2)}=\left(\begin{array}{lll}
0 & 0 & 0 \\
0 & 0 & 0 \\
0 & 0 & 1
\end{array}\right) .
$$

Now according to Born's rule, we can define the probabilities $p_{(2,0)}, p_{(1,1)}$, and $p_{(0,2)}$ and rewrite relations (42) as follows:

$$
\begin{aligned}
& p_{(2,0)}=\operatorname{Tr}\left(\hat{P}_{(2,0)} \hat{\rho}^{3}\right)=\left|C_{1}^{2}\right|^{2}, \\
& p_{(1,1)}=\operatorname{Tr}\left(\hat{P}_{(1,1)} \hat{\rho}^{3}\right)=\left|C_{2}^{2}\right|^{2}, \\
& p_{(0,2)}=\operatorname{Tr}\left(\hat{P}_{(0,2)} \hat{\rho}^{3}\right)=\left|C_{3}^{2}\right|^{2}=\frac{1}{2}\left(1-\left|C_{3}^{1}\right|^{2}\right) .
\end{aligned}
$$

Born's rule is valid for both pure and mixed states; in the case of pure states, formula (42) reads

$$
p\left(x_{i}\right)=\left|\left\langle\Psi_{i} \mid \Psi\right\rangle\right|^{2},
$$

where $\Psi_{i}$ is the basis vector corresponding to the measurement outcome $x_{i}$.

In the case of qutrit-state vector (38), the basis vectors are given by (43), and relations (45) and (46) take the form

$$
\begin{aligned}
& p_{(2,0)}=\left|\left\langle 2,0 \mid \Psi^{(3)}\right\rangle\right|^{2}=\left|C_{1}^{2}\right|^{2} \\
& p_{(1,1)}=\left|\left\langle 1,1 \mid \Psi^{(3)}\right\rangle\right|^{2}=\left|C_{2}^{2}\right|^{2} \\
& p_{(0,2)}=\left|\left\langle 0,2 \mid \Psi^{(3)}\right\rangle\right|^{2}=\left|C_{3}^{2}\right|^{2}=\frac{1}{2}\left(1-\left|C_{3}^{1}\right|^{2}\right) .
\end{aligned}
$$

Here, vectors $\langle 2,0|,\langle 1,1|$, and $\langle 0,2|$ determine the projectors similar to $\hat{P}_{(2,0)}, \hat{P}_{(1,1)}$, and $\hat{P}_{(0,2)}$. Formulas (44) provide the matrix form of these projectors. We see that the real nonnegative numbers $p_{3}^{(22)}$ and $p_{3}^{(33)}$, appearing as traces of products of the density matrix (40) with corresponding dequantizers, are probabilities (45) generated by projectors (44). These probabilities determine the structure of the state (38); such a construction can be generalized to an arbitrary qudit.

In the SPDC process, the other experimental condition, where photons with the same polarization have different frequencies, can take place, namely photons with the horizontal polarization have both frequency $\omega_{s}$ and frequency $\omega_{i}$. Similarly, photons with the vertical polarization also have both frequency $\omega_{s}$ and frequency $\omega_{i}$. In this case, the biphoton wave function describes a ququart state with the state vector

$$
\begin{aligned}
\left|\Psi^{(4)}\right\rangle= & C_{1}\left|1_{H, \omega_{i}}, 1_{H, \omega_{s}}\right\rangle+C_{2}\left|1_{H, \omega_{i}} 1_{V, \omega_{s}}\right\rangle+C_{3}\left|1_{V, \omega_{i}}, 1_{H, \omega_{s}}\right\rangle+C_{4}\left|1_{V, \omega_{i}}, 1_{V, \omega_{s}}\right\rangle \\
= & \left(C_{1} \hat{a}_{H, \omega_{i}}^{+} \hat{a}_{H, \omega_{s}}^{+}+C_{2} \hat{a}_{H, \omega_{i}}^{+} \hat{a}_{V, \omega_{s}}^{+}+C_{3} \hat{a}_{V, \omega_{i}}^{+} \hat{a}_{H, \omega_{s}}^{+}+C_{4} \hat{a}_{V, \omega_{i}}^{+} \hat{a}_{V, \omega_{s}}^{+}\right)|0\rangle, \\
& \left|C_{1}\right|^{2}+\left|C_{2}\right|^{2}+\left|C_{3}\right|^{2}+\left|C_{4}\right|^{2}=1 .
\end{aligned}
$$

Now we consider the example of creating a ququart state. The density matrix (31) completely determines an arbitrary pure ququart state. Thus, in order to reconstruct the 
unknown ququart state, one should measure all its matrix elements; the setup used for this purpose can be found in [36], where the pump radiation of a He-Cd laser with a wavelength of $325 \mathrm{~nm}$ and the horizontal polarization falls on a Lithium Iodate crystal $\mathrm{LiIO}_{3}$, and a biphoton emerges from this nonlinear crystal. It was shown in $[36,55]$ that biphoton state created in this process is the ququart with the coefficients

$C_{1}=-0.0555-0.204 i, \quad C_{2}=-0.0059+0.005 i, \quad C_{3}=-0.0425+0.0052 i, \quad C_{4}=+0.9973$.

Here, $H$ and $V$ are the designations of the polarization modes in the vertical-horizontal laboratory polarization basis.

\section{Conclusions}

To conclude, we formulate the main results of our study.

We presented the probability representation of qudit states and found their quantizers and dequantizers in explicit forms. Examples of qutrit and ququart systems were discussed. The density matrix elements of qudit states were expressed in terms of probabilities, which can be experimentally measured. We suggested a possibility to analyze the photon states arising in the process of parametric down conversion. In the SPDC process, the qutrit photon states are created; for pure states, the probabilities determining the density matrices of created states were found from the experimental data of $[36,55]$. The generalization of the developed approach to other qudit states was studied.

In this paper, we considered qudits of arbitrary dimension and described the structure of their density operators. We developed the general method for constructing quantizer and dequantizer operators for these density operators and found the symbols of the density operator corresponding to such sets of quantizers and dequantizers. These sets of quantizers and dequantizers can be constructed in various ways. The most interesting sets are quantizer and dequantizer, whose symbols have a physical meaning and are directly measurable quantities. We considered two types of such quantities-average values and probabilities of the values and described them using the example of qutrit and ququart states. In general, the relation of these values to the symbols of the density operator requires additional research.

The formalism developed has important physical applications. It can be used to find various characteristics of complex quantum states. This includes restoring elements of the density matrix of states that occur in various nonlinear processes. We considered such an example of the down-conversion process and will look at other examples later. For the experimentally available qutrit and ququart states, realized as photon states generated in the SPDC process, we obtained the explicit probability representation of these states expressed in terms of measurable probabilities. For the ququart pure state with the state vector (48), the $4 \times 4$ density matrix has matrix elements $\rho_{j k}=C_{j} C_{k}^{*}$, where numbers $C_{j}$ given by Equation (49) are experimentally measured. This means that we have the explicit form of the ququart density matrix.

Our main result here is the probability representation of the obtained photon-state density matrix. Since we know how the density matrix elements $\rho_{j k}$ are expressed in terms of probabilities, we know the probabilities $P_{1,2,3}^{j k}$ for $j=1,2,3,4$, since they are functions of the measured parameters $C_{j}$ of the ququart state of the photons created in the SPDC process. Thus, we showed that the qutrit and ququart states of photons created in the SPDC process and experimentally studied in [55] are completely determined by the probabilities. The set of experimentally obtained probabilities includes complete information on the quantum states of the photons.

According to the approach of constructing the associative algebras and Lie algebras by means of quantizer-dequantizer operators [21,22] for qudit states (25) and (26) and ququart states (32) created in down-conversion processes, one can construct the associative algebras and Lie algebras related to the down-conversion process. This problem is closely related to the ideas and methods of quantum tomography. As part of our approach, we 
plan to look at different variants of quantum tomography from a single point of view and establish a connection between them.

Author Contributions: Conceptualization, V.I.M. and V.A.A.; methodology, P.A. and M.A.M.; software, M.M.; validation, P.A., V.A.A. and M.A.M.; formal analysis, M.M.; investigation, V.A.A.; writing-original draft preparation, V.A.A.; writing—review and editing, M.A.M.; supervision, V.I.M. All authors have read and agreed to the published version of the manuscript.

Funding: This research was supported by the National Research, Development and Innovation Office, Hungary (Projects No. K124351 and No. 2017-1.2.1-NKP-2017-00001 HunQuTech). The project has also been supported by the European Union (Grants No. EFOP-3.6.2-16-2017-00005 and No. EFOP-3.4.3-16-2016-00005).

Institutional Review Board Statement: Not applicable.

Informed Consent Statement: Not applicable.

Data Availability Statement: Not applicable.

Acknowledgments: V.I.M. gratefully acknowledges the partial support of the Tomsk State University Competitiveness Improvement Program for obtaining the results of Section 2. V.I.M. is thankful to the support of the Russian Science Foundation under Grant No. 1971-10091 for obtaining the results of Sections 3 and 5. In section Acknowledgments of [21,28], the Russian Science Foundation Grant should read No. 1971-10091 not No. 1871-10091.

Conflicts of Interest: The authors declare no conflict of interest.

\section{References}

1. Schrödinger, E. Quantisierung als Eigenwertproblem (Erste Mitteilung). Ann. Phys. 1926, 384, 361-376. [CrossRef]

2. Schrödinger, E. Quantisierung als Eigenwertproblem (Zweite Mitteilung). Ann. Phys. 1926, 384, 489-527. [CrossRef]

3. Landau, L. Das Dämpfungsproblem in der Wellenmechanik. Z. Phys. 1927, 45, 430-441. [CrossRef]

4. von Neumann, J. Wahrscheinlichkeitstheoretischer Aufbau der Quantenmechanik. Nachr. Ges. Wiss. Göttingen Math. Phys. Kl. 1918, 1918, 98-100.

5. Wigner, E. On the quantum correction for thermodynamic equilibrium. Phys. Rev. 1932, 40, 749-759. [CrossRef]

6. Husimi, K. Some formal properties of the density matrix. Proc. Phys. Math. Soc. Jpn. 1940, 22, 264-314. [CrossRef]

7. Kano, Y. Theory of the electromagnetic field in the statistical theory of the electromagnetic field. J. Math. Phys. 1965, 6, 1913-1915. [CrossRef]

8. Glauber, R.J. Coherent and incoherent states of the radiation field. Phys. Rev. 1963, 131, 2766-2788. [CrossRef]

9. Sudarshan, E.C.G. Equivalence of semiclassical and quantum-mechanical descriptions of statistical light beams. Phys. Rev. Lett. 1963, 10, 277-279. [CrossRef]

10. Avanesov, A.S.; Man'ko, V.I. Dissipative evolution of the qubit State in the tomographic-probability representation. J. Russ. Laser Res. 2017, 38, 311-323. [CrossRef]

11. Klimov, A.B.; Romero, J.L.; Bjork, G.; Sanchez-Soto, L. Geometrical approach to mutually unbiased bases. J. Phys. A Math. Theor. 2007, 40, 3987-3998. [CrossRef]

12. Mancini, S.; Man'ko, V.I.; Tombesi, P. Symplectic tomography as classical approach to quantum systems. Phys. Lett. A 1996, 213, 1-6. [CrossRef]

13. Dodonov, V.V.; Man'ko, V.I. Positive distribution description for spin states. Phys. Lett. A 1997, 229, 335-339. [CrossRef]

14. Man'ko, V.I.; Man'ko, O.V. Spin state tomography. J. Exp. Theor. Phys. 1997, 85, 430-434. [CrossRef]

15. Adam, P.; Andreev, V.A.; Man'ko, M.A.; Man'ko, V.I. Nonnegative discrete symbols and their probabilistic interpretation. J. Russ. Laser Res. 2017, 38, 491-506. [CrossRef]

16. D'Ariano, G.M.; Maccone, L.; Paini, M. Spin tomography. J. Opt. B Quantum Semiclass. Opt. 2003, 5, 77-84. [CrossRef]

17. Terra-Cunha, M.O.; Man'ko, V.I.; Scully, M.O. Quasiprobability and probability distributions for spin-1/2 states. Found. Phys. Lett. 2001, 14, 103-117. [CrossRef]

18. Chernega, V.N.; Man'ko, O.V.; Man'ko, V.I. Probability representation of quantum observables and quantum states. J. Russ. Laser Res. 2017, 38, 324-333. [CrossRef]

19. Asorey, M.; Ibort, A.; Marmo, G.; Ventriglia, F. Quantum tomography twenty years later. Phys. Scr. 2015, 90, 074031. [CrossRef]

20. Man'ko, M.A.; Man'ko, V.I. From quantum carpets to quantum suprematism-the probability representation of qudit states and hidden correlations. Phys. Scr. 2018, 93, 084002. [CrossRef]

21. Adam, P.; Andreev, V.A.; Man'ko, M.A.; Man'ko, V.I.; Mechler, M. SU(2) Symmetry of Qubit States and Heisenberg-Weyl Symmetry of Systems with Continuous Variables in the Probability Representation of Quantum Mechanics. Symmetry 2020, 12, 1099. [CrossRef] 
22. Andreev, V.A.; Man'ko, M.A.; Man'ko, V.I. Quantizer-dequantizer operators as a tool for formulating the quantization procedure. Phys. Lett. A 2020, 384, 126349. [CrossRef]

23. Doskoch, I.Y.; Man'ko, M.A. Hidden correlations and information-entropic inequalities in systems of qudits. J. Russ. Laser Res. 2019, 40, 293-312. [CrossRef]

24. Doskoch, I.Y.; Man'ko, M.A. Superposition principle and Born's rule in the probability representation of quantum states. Quantum Rep. 2019, 1, 130-150. [CrossRef]

25. Man'ko, M.A.; Man'ko, V.I.; Marmo, G.; Ventriglia, F.; Vitale, P. Dichotomic probability representation of quantum states. arXiv 2019, arXiv:1905.10561.

26. Doskoch, I.Y.; Man'ko, M.A. New correlation relations in classical and quantum systems with different numbers of subsystems. J. Phys. Conf. Ser. 2020, 1612, 012011. [CrossRef]

27. Adam, P.; Andreev, V.A.; Man'ko, M.A.; Man'ko, V.I.; Mechler, M. Star-product formalism for the probability and mean-value representations of qudits. J. Russ. Laser Res. 2020, 41, 470-483. [CrossRef]

28. Chernega, V.N.; Man'ko, M.A.; Man'ko, V.I. $\mathcal{P} \mathcal{T}$-symmetric qubit-system states in the probability representation of quantum mechanics. Symmetry 2020, 12, 1702. [CrossRef]

29. Kiktenko, E.O.; Malyshev, A.O.; Mastiukova, A.S.; Man'ko, V.I.; Fedorov, A.K.; Chruscinski, D. Probability representation of quantum dynamics using pseudostochastic maps. Phys. Rev. A 2020, 101, 052320. [CrossRef]

30. Chruscinski, D.; Marmo, G.; Man'ko, V.I.; Ventriglia, F. On pseudo-stochastic matrices and pseudo-positive maps. Phys. Scr. 2015, 90, 115202. [CrossRef]

31. Man'ko, M.A.; Man'ko, V.I. Observables, interference phenomenon and Born's rule in the probability representation of quantum mechanics. Int. J. Quantum Inf. 2020, 18, 1941021. [CrossRef]

32. Chernega, V.N.; Man'ko, M.A.; Man'ko, V.I. Schroedinger equation for energy levels as a linear equation for probability distributions identified with quantum states. J. Russ. Laser Res. 2020, 41, 441-450. [CrossRef]

33. Chernega, V.N.; Man'ko, M.A.; Man'ko, V.I. Quantized-energy equation for N-level atom in the probability representation of quantum mechanics. J. Russ. Laser Res. 2020, 41, 576-583. [CrossRef]

34. Adam, P.; Andreev, V.A.; Man'ko, M.A.; Man'ko, V.I. Symbols of multiqubit states admitting a physical interpretation. J. Russ. Laser Res. 2018, 39, 360-375. [CrossRef]

35. Laptev, G.D.; Novikov, A.A.; Chirkin, A.S. Interaction of light waves in active nonlinear and periodically poled nonlinear crystals. JETP Lett. 2003, 78, 38-50. [CrossRef]

36. Bogdanov, Y.I.; Galeev, R.F.; Kulik, S.P.; Maslennikov, G.A.; Moreva, E.V. Reconstruction of the polarization states of a biphoton field. JETP Lett. 2005, 82, 164-168. [CrossRef]

37. Man'ko, O.V.; Man'ko, V.I.; Marmo, G. Alternative commutation relations, star products and tomography. J. Phys. A Math. Gen. 2002, 35, 699-719. [CrossRef]

38. Lizzi, F.; Vitale, P. Matrix bases for star products: A review. SIGMA 2014, 10, 086. [CrossRef]

39. Adam, P.; Andreev, V.A.; Isar, A.; Man'ko, M.A.; Man'ko, V.I. Continuous sets of dequantizers and quantizers for one-qubit states. J. Russ. Laser Res. 2016, 37, 544-555. [CrossRef]

40. Ciaglia, F.M.; Di Cosmo, F.; Ibort, A.; Marmo, G. Dynamical aspects in the quantizer-dequantizer formalism. Ann. Phys. 2017, 385, 769-781. [CrossRef]

41. Man'ko, V.I.; Markovich, L.A. Integral transforms between tomogram and quasiprobability functions based on quantizerdequantizer operator formalism. J. Math. Phys. 2020, 61, 102102. [CrossRef]

42. Stratonovich, P.L. On distributions in representation space. Sov. Phys. J. Exp. Theor. Phys. 1957, 4, 891-898.

43. Sudarshan, E.C.G. Search for purity and entanglement. J. Russ. Laser Res. 2003, 24, 195-203. [CrossRef]

44. Man'ko, M.A. Information and entropic characteristics of photon and qudit quantum states. Phys. Scr. 2010, T40, 014027. [CrossRef]

45. Chernega, V.N.; Man'ko, O.V. Generalized qubit portrait of the qutrit-state density matrix. J. Russ. Laser Res. 2013, 34, 383-387. [CrossRef]

46. Chernega, V.N.; Man'ko, O.V.; Man'ko, V.I. Triangle geometry for qutrit states in the probability representation. J. Russ. Laser Res. 2017, 38, 416-425. [CrossRef]

47. Rodionov, A.V.; Chirkin, A.S. Entangled photon states in consecutive nonlinear optical interactions. JETP Lett. 2004, 79, $253-256$. [CrossRef]

48. Wei, B.; Cai, W.-H.; Ding, C.; Deng, G.-W.; Shimizu, R.; Zhou, Q.; Jin, R.-B. Mid-infrared spectrally-uncorrelated biphoton generation from doped PPLN: A theoretical investigation. Opt. Express 2020, 29, 256-271. [CrossRef]

49. Fedorov, M.V.; Volkov, P.A.; Mikhailova, J.M.; Straupe, S.S.; Kulik, S.P. Entanglement of biphoton states: Qutrits and ququarts. New J. Phys. 2011, 13, 083004. [CrossRef]

50. Chekhova, M.V.; Fedorov, M.V. The Schmidt modes of biphoton qutrits: Poincare-sphere representation. J. Phys. B At. Mol. Opt. Phys. 2013, 46, 095502. [CrossRef]

51. Andreev, V.A.; Ivanova, O.A. The dynamics of three-boson interaction and algebraic Bethe ansatz. Phys. Lett. A 1992, 171, 145-150. [CrossRef]

52. Andreev, V.A.; Ivanova, O.A. Equations for Bethe ansatz parameters and parametric surfaces in three-boson interaction. Phys. Lett. A 1996, 216, 76-86. [CrossRef] 
53. Andreev, V.A.; Ivanova, O.A. Symmetries and reduced system of equations for three-boson and four-boson interactions. J. Phys. A Math. Gen. 2002, 35, 8587-8602. [CrossRef]

54. Gleason, A.M. Measures on the closed subspaces of a Hilbert space. Indiana Univ. Math. J. 1957, 6, 885-893. [CrossRef]

55. Moreva, E.V.; Maslennikov, G.A.; Straupe, S.S.; Kulik, S.P. Realization of four-level qudits using biphotons. Phys. Rev. Lett. 2006, 97, 023602. [CrossRef] 